\title{
Welfare and Convergence Speed in the Ramsey Model under Two Classes of Gorman Preferences
}

\author{
Wilson da Cruz Vieira ${ }^{1}$ Alberto Bucci $^{2} \cdot$ Simone Marsiglio $^{3}$
}

\begin{abstract}
Using a one-sector, discrete-time Ramsey model, we analyze and compare the implications for welfare, capital accumulation, and speed of convergence to the steady state of two classes of utility functions that represent Gorman preferences, namely homothetic and Stone-Geary preferences. For identical economies, we show that the preference structure does not affect only the capital dynamics and social welfare but also the speed of convergence to the steady-state equilibrium.
\end{abstract}

Keywords Ramsey Model • Preferences $•$ Convergence Speed $\cdot$ Social Welfare JEL Classification C61 $\cdot \mathrm{E} 13 \cdot \mathrm{O} 41 \cdot \mathrm{O} 47$

\section{Introduction}

In this paper we analyze the role of preferences in a standard neoclassical Ramsey framework, which represents the workhorse model of modern macroeconomic theory. This is a perfectly competitive general equilibrium setup in which the utility function representing consumer preferences characterizes the economy's demand side while the production function describing producers' behavior the supply side. Focusing on the demand side, our main objective is to investigate, using a one-sector discrete time framework, the implications for welfare, capital accumulation and convergence speed to the steady-state of two classes of utility functions that represent Gorman preferences, namely homothetic and Stone-Geary preferences.

\footnotetext{
${ }^{1}$ Corresponding author. Department of Agricultural Economics, Federal University of Viçosa, Brazil. Email: wvieira@ufv.br

${ }^{2}$ Department of Economics, Management and Quantitative Methods, University of Milan, Italy

${ }^{3}$ Department of Economics and Management, University of Pisa, Italy
} 
As is well known, a key condition characterizing the optimal solution of the onesector Ramsey model is that the gross marginal productivity of capital equals the gross discount rate at the steady-state, that is, $f^{\prime}\left(k^{s s}\right)=\frac{1}{\beta}$, where $f(\cdot)$ is the production function and $\beta$, the discount factor. Both steady-state capital stock and consumption do not depend on preferences, although preferences may affect the transition dynamics of these variables to the steady state. Sundaram (1996, p. 300), for example, states for this model that the stationary states of capital stock and consumption are “...independent of the properties of the utility function, and depend only on the properties of the technology $f(\cdot)$ and the discount factor $\beta$ ". This author emphasizes that this is true only for the steady state values of the capital stock and consumption and, in his footnote 5 (p. 300), he admits that the trajectories by which the stock of capital and consumption converge to their stationary states "...do depend on the utility function”.

Based on these facts, the main motivation for investigating the role of preferences on welfare, capital dynamics, and speed of convergence to the steady state is twofold: first, criticisms of the traditional use of homothetic preferences in growth models (see, for example, Matsuyama 1992); and second, there is great interest in empirical studies on convergence between economies (see, for example, Barro and Sala-i-Martin 1992). In addition, both homothetic and Stone-Geary (non-homothetic) preferences are widely used in economic analysis and, to the best of our knowledge, there is no previous work comparing their implications under the Ramsey framework.

The Engel curves are straight lines for goods with Marshallian demand functions generated from utility functions that represent these types of preferences (homothetic and Stone-Geary preferences). Both types of preferences, homothetic and Stone-Geary, are part of a larger class of preferences called Gorman preferences, that is, preferences that admit indirect utility functions of the Gorman form. In economies with Gorman preferences the quantities demanded can be aggregated, that is, the aggregate variable does not depend on income distribution and we can treat aggregate demand as if it were generated by a representative consumer. ${ }^{4}$ In this sense, for a one-sector economy, we can rely on the welfare changes of this fictitious individual consumer as a measure of social welfare, that is, a measure of well-being of the whole economy.

Despite its analytical convenience, the use of homothetic preferences in economic growth models has received a number of criticisms. One of these is related to the fact

\footnotetext{
${ }^{4}$ See, for example, Chipman (2006) for a discussion on aggregation in demand theory.
} 
that this class of preferences implies income elasticity of demand equal to one for all types of goods (see, for example, Matsuyama 1992), while there is empirical evidence that food and luxury goods, for example, have income elasticity of demand different from one. Another critique concerns the constant intertemporal elasticity of substitution of homothetic preferences. Chari and Kehoe (2006), for example, attribute the policy arguments against capital taxation to the homotheticity of preferences over consumption at different periods. In order to overcome these criticisms, several papers have adopted non-homothetic specification for preferences.

Stone-Geary preferences are a classic example of non-homothetic preferences widely used in the economic literature. This class of preferences has been used in recent growth literature to address several issues, both theoretical and empirical. ${ }^{5}$ Unlike ours, some of these works seek to analyze the effects on economic growth of structural changes in models with two or more sectors, such as Kongsamut et al. (2001), Irz and Roe (2005), and Matsuyama (1992). Kongsamut et al. (2001) develop a three-sector standard growth model with utility specification embedding different income elasticities consistent with both the Kaldor facts and the dynamics of sectoral labor reallocation. Irz and Roe (2005) use a two-sector Ramsey model with utility assumed to take the StoneGeary form to investigate the relationship between agricultural productivity and economy-wide growth, showing that the level and growth rate of agricultural productivity influence the speed of capital accumulation. Matsuyama (1992) addresses the role of agricultural productivity in a two-sector growth model in which preferences are of Stone-Geary class and the engine of growth is learning-by-doing in the manufacturing sector.

Authors who work with one-sector growth models and Stone-Geary preferences, but with goals different from ours, include, for example, Álvarez-Peláez and Díaz (2005), Chatterjee and Ravikumar (1999), and Steger (2000). Álvarez-Peláez and Díaz (2005) investigate quantitatively the effects of economic growth on inequality focusing on the importance of differences in saving rates across households. Chatterjee and Ravikumar (1999), in turn, study the effects of a minimum consumption requirement on both economic growth rate and evolution of wealth distribution. Steger (2000) is the closest in spirit to our work, because it addresses both the transition path and convergence in his analysis. However, unlike ours, Steger (2000) uses a linear model of

\footnotetext{
${ }^{5}$ For empirical evidence on non-constant intertemporal elasticity of substitution, see, for example, Atkeson and Ogaki (1996) or Crossley and Low (2011).
} 
endogenous growth and approaches convergence differently. ${ }^{6}$ We notice that none of these works compare the implications of different preferences structures (homothetic versus Stone-Geary preferences) in a way comparable to ours.

Regarding the speed of convergence between economies, there is an extensive empirical literature on this topic (see, for example, Barro and Sala-i-Martin 1992; and de la Fuente 1997). A recurring hypothesis in these empirical works is to test whether less developed economies grow at faster rates than developed economies. Unlike this literature, we take a theoretical approach to the speed of convergence between economies. In this sense, the main contribution of this paper is thus to clarify and to show theoretically how different classes of Gorman preferences (homothetic and StoneGeary preferences) may affect the dynamics of capital, social welfare as well as the convergence speed to the steady-state in economies with identical structures.

In order to isolate and compare the effects of these two classes of Gorman preferences, we consider one-sector economies in which there is no uncertainty sharing the same characteristics (that is, same production technology and same market structure, same initial capital stock and discount factor), and differing solely with respect to the preferences structure. Using general specifications for utility functions that admit income elasticity of demand equal to or less than one and a set of fairly mild assumptions, we show that: i) the optimal level of savings (capital stock) under homothetic preferences is at least as large as the optimal level of savings under StoneGeary preferences; ii) the social welfare is strictly higher under homothetic preferences; and iii) economies with homothetic preferences converge more quickly to the steady state when compared with economies with Stone-Geary preferences.

The paper proceeds as follows. Section 2 presents the model and the assumptions used throughout the paper. In section 3 we analyze the role of preferences on capital dynamics, convergence speed to the steady-state, and social welfare, deriving our main results. Finally, in section 4 , as usual, we present some concluding remarks and propose directions for future research.

\footnotetext{
${ }^{6}$ We analyze global convergence in an exogenous one-sector growth model from the linearization of the policy function around the steady state while Steger (2000) analyzes convergence/divergence in a context of endogenous growth.
} 


\section{Model and Assumptions}

\subsection{The model}

We consider two closed economies that differ only with respect to their preferences and that are inhabited by consumers who live forever. We denote by $N_{t}$ the same number of consumers of both economies at each period $t$. The population of these economies, by assumption, grows at the same rate $n$, that is, $N_{t}=(1+n)^{t} N_{0}$, where $n=\Delta N_{t+1} / N_{t}$ and $N_{0}$ is the same number of consumers of both economies in period 0 . There is a single good in both economies that may be consumed or invested. The consumption of one consumer, at each period $t$, is denoted by $c_{t}$. The representative consumer of each economy wants to maximize the total utility over her lifetime, that is,

$$
\max _{0} \sum_{t=0}^{\infty} \beta^{t}(1+n)^{t} u_{j}\left(c_{t}\right), j=a, b,
$$

where $\beta \in(0,1)$ is the discount factor and $u_{j}: R_{+} \rightarrow R, j=a, b$, is the instantaneous utility function. The subscript $j$ refers to the preference type, with " $a$ " being StoneGeary preferences and " $b$ ", homothetic preferences. At each period $t$, the level of consumption $c_{t}$ of each economy is subject to the constraint:

$$
N_{t} c_{t}+I_{t} \leq F\left(K_{t}, N_{t}\right)
$$

where $I_{t}$ is the total investment at each period $t, F$ is the aggregate production function, $K_{t}$ is the total capital stock at each period $t$, and $N_{t}$ is the number of workers at each period $t$ (here we are assuming that consumers and workers are physically identical and that the entire population of both economies work). The capital accumulation in both economies is given by the equation:

$$
K_{t+1}=K_{t}(1-\delta)+I_{t},
$$

where $\delta \in(0,1]$ is the depreciation rate of the capital stock. Making the assumption that the production function exhibits constant returns to scale and letting $k_{t}=K_{t} / N_{t}$ be the capital stock in per capita values, the constraint between consumption and investment for both economies, for each period $t$, becomes:

$$
c_{t}+k_{t+1}(1+n) \leq F\left(k_{t}, 1\right)+(1-\delta) k_{t} .
$$

Assuming, for simplicity, that $N_{0}=1, n=0, \delta=1$, and defining the function $f$ by $f\left(k_{t}\right)=F\left(k_{t}, 1\right)$, the Ramsey growth model for both economies will have the following compact form:

$$
\max \sum_{t=0}^{\infty} \beta^{t} u_{j}\left(c_{t}\right), j=a, b
$$


subject to the constraints:

$$
\begin{aligned}
& c_{t}+k_{t+1} \leq f\left(k_{t}\right), \forall t, \\
& c_{t} \geq 0, k_{t} \geq 0, \forall t,
\end{aligned}
$$

and $k_{0} \geq 0$ is given. As defined above, the subscript $j$ refers to the preference type, with " $a$ " being Stone-Geary preferences and " $b "$, homothetic preferences.

As mentioned in the Introduction, we consider two classes of Gorman preferences, homothetic and Stone-Geary (non-homothetic), that is, classes of preferences in which each individual's utility function has an indirect utility function of the Gorman form. These preferences imply linear Engel curves and are characterized by an aggregation property such that the consumption that emerges by aggregating individual consumption of agents with different incomes can be treated as if it were generated by a representative agent. Although aggregate consumption does not depend on income distribution, it depends on income levels if preferences are quasi-homothetic such as those of Stone-Geary and, in such case, this implies that the marginal propensity to consume is not constant along transition paths. ${ }^{7}$ We adopt the following general specification for utility functions that represent these preferences:
(a) $u_{a}\left(c_{t}\right):[\bar{c}, \infty) \rightarrow R$,$$
u_{a}=u_{a}\left(c_{t}-\bar{c}\right), \quad \bar{c}>0
$$
(Stone-Geary)
(b) $u_{b}\left(c_{t}\right):[0, \infty) \rightarrow R$,
$u_{b}=u_{b}\left(c_{t}\right)$,
$\bar{c}=0$
(Homothetic)

Note that, when we have $\bar{c}>0$, the utility function $u_{a}\left(c_{t}\right):[\bar{c}, \infty) \rightarrow R, u_{a}=$ $u_{a}\left(c_{t}-\bar{c}\right)$, is really non-homothetic because, in this case, it is impossible to find a monotone transformation of a homogeneous function and get as a result a homothetic utility function. This positive parameter also implies that the intertemporal elasticity of substitution in period $t$ is no longer a constant. There are two classic types of restrictions that we can impose on preference relations in order to get indirect utility functions of the Gorman form: homotheticity and quasi-linearity. Recall that a preference relation is homothetic if the slope of indifference curves along any ray from the origin remains constant; all indifference curves have the same shape because they are "radial blow ups" of each other. On the other hand, a preference relation is quasilinear if there is one good, called the numeraire, which shifts the indifference curves outward as consumption of it increases, without changing their shape. ${ }^{8}$

\footnotetext{
${ }^{7} \mathrm{We}$ are grateful to one of the reviewers who drew our attention on this point. A more detailed discussion of this issue can be found, for example, in Bertola et al. (2005), or Pollak (1971).

${ }^{8}$ For more details on homothetic and quasilinear preferences and their respective representations as utility functions, see, for example, Rubinstein (2012).
} 
Classic examples of utility functions that represent homothetic preferences include the constant elasticity of substitution (CES) utility function and those that are derived from it, such as the Cobb-Douglas, logarithm and linear utility functions. StoneGeary utility functions can be obtained from both homothetic and quasilinear preferences. ${ }^{9}$ For example, in order to define them from homothetic preferences, suppose there are $J$ goods and let $s=\left(s_{1}, \ldots, s_{J}\right)$ be a vector of $J$ parameters, which may be either positive, negative or zero. Stone-Geary preferences can be represented by the following utility function:

$$
u\left(x_{1}, \ldots, x_{J}\right)=\prod_{j=1}^{J}\left(x_{j}-s_{j}\right)^{\alpha_{j}},
$$

where $\alpha_{j}>0$ for all $j$ and $\sum_{j=1}^{J} \alpha_{j}=1$. Note that this utility function is a simple extension of the Cobb-Douglas utility function. We can think of Stone-Geary preferences as simply Cobb-Douglas preferences expressed relative to the vector of parameters $s=\left(s_{1}, \ldots, s_{J}\right)$ rather than relative to the usual origin. Under this perspective, the Cobb-Douglas utility function is a special case of the Stone-Geary utility function when the terms $s_{1}, \ldots, s_{J}$ are all equal to zero.

Since we consider economies with a single sector that produces a single good that can be consumed or invested, the Stone-Geary utility function could take, for example, the following functional form: $u(c)=(c-\bar{c})^{1-\sigma} /(1-\sigma)$, where $c \geq \bar{c} \geq 0$ denotes consumption and $\sigma>0$, or $u(c)=\sqrt{c-\bar{c}}$, where $c \geq \bar{c} \geq 0$. If $\bar{c}=0$, we obtain the associated homothetic utility functions. ${ }^{10}$ In order to get results as general as possible, we work with general functional form for both the utility functions and the production function. It is only required that these functions meet certain conditions, such as continuity, convexity, differentiability and Inada conditions. However, we illustrate the main results obtained with numerical examples that use specific functional forms for both utility functions and production function.

\footnotetext{
${ }^{9}$ It is possible to obtain a Stone-Geary utility function from a positive monotone transformation of a quasilinear utility function. For example, let the quasilinear utility function $u\left(x_{1}, x_{2}\right)=v\left(x_{1}\right)-x_{2}$, where $x_{2}$ is the numeraire and $v\left(x_{1}\right)$ is a concave function. Fix the numeraire $x_{2}=\bar{s}$ and let $v\left(x_{1}\right)=x$. Now, the quasilinear utility function is given by $u(x, \bar{s})=x-\bar{s}$. By doing $w(x, \bar{s})=\log (x-\bar{s})$ we get as a result a Stone-Geary utility function.

${ }^{10}$ Since we assume that the utility functions satisfy the Inada conditions (see Assumption 4), we consider only non-negative values for the parameter $\bar{c}$.
} 


\subsection{The Assumptions}

We present below a set of assumptions that the preferences and production function must satisfy and whose main objective is not only to guarantee the existence of solution to the intertemporal optimization problem (1) - (3) but also to allow the characterization of this solution. These assumptions are standards in the economic literature of economic growth and can be found in textbooks such as Sundaram (1996) and Stokey and Lucas with Prescott (1989). The basic difference here, compared to textbooks, is the introduction of the $\bar{c} \geq 0$ parameter in the model structure.

In this sense, to avoid internal inconsistencies in the models analyzed, we assume from now on and in the rest of this paper that $k_{0} \geq \bar{c} \geq 0$ and $\bar{c} \leq k^{s s}$, where $k^{s s}>0$ is the steady state capital stock. The basic assumptions related to the utility functions and production function are as follows:

Assumption 1 For $j=a, b, u_{j}\left(c_{t}\right)$ is continuous in its domain and is strictly increasing with $u_{a}(\bar{c})=0$ and $u_{b}(0)=0$.

If Assumption 1 is satisfied, at the optimum, the constraints will be binding. Thus, the initial intertemporal optimization problem (1) - (3) is equivalent to the following:

$$
\max \sum_{t=0}^{\infty} \beta^{t} u_{j}\left(f\left(k_{t}\right)-k_{t+1}-\bar{c}\right), \beta \in(0,1), j=a, b
$$

subject to the constraints:

$$
0 \leq \bar{c} \leq k_{t+1} \leq f\left(k_{t}\right), \forall t,
$$

and $k_{0} \geq \bar{c} \geq 0$ is given. As defined above, the subscript $j$ refers to the preference type, with " $a$ " being Stone-Geary preferences $(\bar{c}>0)$ and " $b$ ", homothetic preferences $(\bar{c}=0)$. Note that only consumption exceeding the minimum level, $\bar{c}>0$, generates utility for consumers with preferences of group (a).

We say that an infinite sequence of capital stocks $k=\left(k_{0}, k_{1}, \ldots, k_{t}, \ldots\right)$ is feasible from $k_{0} \geq \bar{c} \geq 0$ if it satisfies:

$$
0 \leq \bar{c} \leq k_{t+1} \leq f\left(k_{t}\right), \forall t .
$$

Assumption 2 The production function $f$ is continuous and non-decreasing in its domain with $f(0)=0$, and there is $\bar{k}>0$ such that $f(k) \leq k$ for all $k \geq \bar{k}$. 
This characterization of the production process is used, for example, in Sundaram (1996, p. 301). The final part of Assumption 2 is to be understood as some sort of decreasing marginal return. For simplicity, we assume that there is no technical progress and that the technology is identical for both economies (with Stone-Geary and homothetic preferences). Based on Assumptions 1 and 2 one can prove that the intertemporal optimization problem (4) - (5) has solution, that is, there are optimal trajectories for both capital stock and consumption that satisfy the constraints imposed on the model. ${ }^{11}$

Let $k=\left(k_{0}, k_{1}, \ldots, k_{t}, \ldots\right)$ be the optimal sequence of capital stocks from $k_{0} \geq \bar{c} \geq 0$ of the intertemporal optimization problem (4) - (5). The associated optimal consumption path $c$ is given by $c_{t}=f\left(k_{t}\right)-k_{t+1}-\bar{c}, \forall t$, with $\bar{c}=0$ for the economy with homothetic preferences. From the optimal sequence of capital stocks we can define the following value function:

$$
v^{j}\left(k_{0}\right)=\max \sum_{t=0}^{+\infty} \beta^{t} u_{j}\left(f\left(k_{t}\right)-k_{t+1}-\bar{c}\right), j=a, b,
$$

with $\bar{c}>0$ for Stone-Geary preferences $(j=a)$ and $\bar{c}=0$ for homothetic preferences $(j=b)$. According to this definition, the value function represents the maximized value of discounted utility flows from consumption over the planning horizon (from zero to infinity) subject to the constraint set.

As is well known, the value function satisfies the Bellman equation:

$$
v^{j}(k)=\max _{k^{\prime} \in[\bar{c}, f(k)]}\left\{u_{j}\left[f(k)-k^{\prime}-\bar{c}\right]+\beta v^{j}\left(k^{\prime}\right)\right\}, j=a, b,
$$

with $\bar{c}=0$ for homothetic preferences and $\bar{c}>0$ for Stone-Geary preferences.

The next assumptions, although restrictive, allow a detailed characterization of the optimal solution of the intertemporal optimization problem (4) - (5). In order to get some result on the effects of alternative preferences on capital dynamics, convergence speed to the steady-state and social welfare we have to impose some additional restrictions on preferences. Considering that that there are many possibilities for functional forms for both homothetic and Stone-Geary preferences and the necessity to make comparisons, we adopt the following restrictive assumption.

\footnotetext{
${ }^{11}$ For proof of the existence of optimal trajectories of consumption and capital stock for the intertemporal optimization problem (4) - (5), see, for example, Sundaram (1996) or Stokey and Lucas with Prescott (1989).
} 
Assumption 3 The utility functions $u_{j}\left(c_{t}\right)=u_{j}\left(c_{t}-\bar{c}\right), j=a, b$, and $c_{t} \geq \bar{c} \geq 0, \forall t$, are strictly concave in their domain and have the same functional forms, except by the shift parameter $\bar{c}$, where $\bar{c}>0$ for $j=a$ and $\bar{c}=0$ for $j=b$.

This assumption is to be understood as follows: if the utility function that represents homothetic preferences is given, for example, by $u_{b}\left(c_{t}\right)=\sqrt{c_{t}}, c_{t} \geq 0, \forall t$, the associated utility function that represents Stone-Geary preferences is given by $u_{a}\left(c_{t}\right)=\sqrt{c_{t}-\bar{c}}, c_{t} \geq \bar{c}>0, \forall t$. As will be seen just below, this assumption is fundamental to understand the differences imposed on the optimal solution in response to changes in the structure of preferences. Sundaram (1996) shows, under Assumptions $1-3$ and the fact that the utility function is strictly concave in its domain (part of Assumption 3), that the optimal path of capital stock is monotonic and unique.

The next two assumptions complete the set of assumptions that are necessary not only to guarantee the existence and characterize the optimal solution of the standard model of economic growth, but also to derive our main results.

Assumption 4 The utility functions $u_{j}\left(c_{t}\right)=u_{j}\left(c_{t}-\bar{c}\right), j=a, b$, are $C^{2}$ in the interior of their domain with $u_{a}^{\prime}(\bar{c})=\infty$ for $j=a$ and $u_{b}^{\prime}(0)=\infty$ for $j=b$.

Assumption 5 The production function is $C^{2}$ in the interior of its domain and is strictly concave on $R_{+}$with $f^{\prime}(0)>1 / \beta$.

The Inada conditions, $u_{a}^{\prime}(\bar{c})=\infty, u_{b}^{\prime}(0)=\infty$, and $f^{\prime}(0)>1 / \beta$, ensure that the optimal solution is interior, that is, the representative consumer will try to maintain strictly positive consumption levels in all periods. This property and those of differentiability of utility and production functions allow characterizing the solution through Euler equations, that is, the optimal solution (sequence of optimal capital stocks) satisfies the following relation:

$$
u_{j}^{\prime}\left(f\left(k_{t}\right)-k_{t+1}-\bar{c}\right)=\beta u_{j}^{\prime}\left(f\left(k_{t+1}\right)-k_{t+2}-\bar{c}\right) f^{\prime}\left(k_{t+1}\right), j=a, b, \forall t .
$$

From the solution of the Bellman equation emerges the policy function $k^{\prime}=$ $g_{j}(k), j=a, b$ (see, for example, Sundaram,1996). Under the above assumptions it can be shown that the value function is continuous, non-decreasing, concave, and $C^{2}$, and that the policy function is continuous, $C^{1}$, and non-decreasing. ${ }^{12}$

\footnotetext{
${ }^{12}$ For more details on the differentiability of both value function and policy function, see, for example, Araujo (1991).
} 
In deriving our main results, we take as given the following results discussed above for the intertemporal optimization problem (4) - (5) obtained under Assumptions 1 - 5. These results are summarized below.

(i) Given $k_{0} \geq \bar{c} \geq 0$, there exists a unique optimal solution to the intertemporal optimization problem (4) - (5), that is, each model $(j=a$ or $j=b$ ) has a single optimal solution.

(ii) The optimal sequences of capital stocks of both economies (with homothetic and Stone-Geary preferences) converge to the same steady state $k^{s s}$, which can be obtained from the expression $\beta f^{\prime}\left(k^{s s}\right)=1$, since both economies have the same technology and same discount factor $\beta$.

(iii) When $k_{0} \geq \bar{c} \geq 0$, the unique optimal solution of each economy (with homothetic or Stone-Geary preferences) is interior and satisfies its associated Euler equation (7).

(iv) The policy function of each economy (with homothetic or Stone-Geary preferences) is continuous, $C^{1}$, and non-decreasing.

\section{Main Results}

The analysis developed below seeks to show that preferences are important in explaining differences between economies with similar structures. That is, if two economies differ only with respect to their consumers' preferences, they may exhibit differences with respect to capital dynamics, convergence speed to the steady-state and social welfare. What is striking about our results is that they are obtained with preferences belonging to the same class, Gorman preferences, that is, preferences that have common properties and differ basically with respect to the importance given to the consumption good, determined by a shift parameter that imposes a minimum consumption requirement.

In our first result we show that if two economies differ only with respect to consumer preferences (one has homothetic preferences and the other Stone-Geary preferences), the optimal trajectories of capital stocks to the single steady state will be distinct. This result is accepted by many authors (see, for example, Sundaram 1996, p. 300), but, as far as we know, there is no formal proof of it comparing these two types of preferences in a standard economic growth model as we do in this paper. This result is 
important because it has implications for both the convergence speed to the single steady-state and the social welfare of these economies.

Proposition 1 Under Assumptions $1-5$, if $k^{s s} \neq k_{0} \geq \bar{c} \geq 0$, the optimal sequences of capital stocks of both economies (with homothetic and Stone-Geary preferences) from $k_{0}$ to the single steady-state capital stock $k^{\text {ss }}$ will be different.

Proof See Appendix A

The main implication of Proposition 1 is that if two economies differ only with respect to consumer preferences (homothetic versus Stone-Geary) their optimal capital stock trajectories to the steady-state will be distinct if $k^{s S} \neq k_{0} \geq \bar{c} \geq 0$. Note that this result is valid even if we assume that $k^{s S}>k_{0} \geq \bar{c} \geq 0$ or $k^{s S}<k_{0} \geq \bar{c} \geq 0$ with $\bar{c} \leq k^{s s}$. These distinct optimal trajectories will be referred to as $k^{a}=\left(k_{0}, k_{1}^{a}, \ldots, k_{t}^{a}, \ldots\right)$, in the case of Stone-Geary preferences, and $k^{b}=$ $\left(k_{0}, k_{1}^{b}, \ldots, k_{t}^{b}, \ldots\right)$, in the case of homothetic preferences. Since we know from Proposition 1 that these trajectories are distinct, we can now investigate other properties of them, such as the convergence speed to the steady state and social welfare of each economy.

The next proposition shows that the preferences also affect the convergence speed to the steady state. Our measure of convergence speed is derived by linearization of the policy function around the steady-state (Ortigueira and Santos 1997), that is, we base our analysis on the policy functions $k^{\prime}=g_{j}(k), j=a, b$, where $j=a$ represents StoneGeary preferences and $j=b$, homothetic preferences. Following Krusell (2004), we use the first derivative of the policy function at the steady-state, $g^{\prime}\left(k^{s S}\right)$, as a measure of the rate of convergence and we use as proxy for this derivative the smallest eigenvalue that emerges as solution of the second order difference equation obtained from the linearization of the Euler equation (7). One parameter of this difference equation, the negative inverse of absolute risk aversion, will allow us to differentiate the convergence rates between economies with Stone-Geary preferences and those with homothetic preferences.

As is well known, the higher the curvature of the utility function the higher the absolute risk aversion (see, for example, Rubinstein 2012). Given the utility function $u(c)$, its risk measure is given by the expression $A(c)=-u^{\prime \prime}(c) / u^{\prime}(c)$. Note that Assumption 3, adopted above, gives a more pronounced curvature for the utility 
function of the economy with Stone-Geary preferences when compared with the utility function of the economy with homothetic preferences. Figure 1 illustrates this fact. Note also that, for low consumption levels, the difference between utility levels is larger and tends to narrow as consumption levels increase.

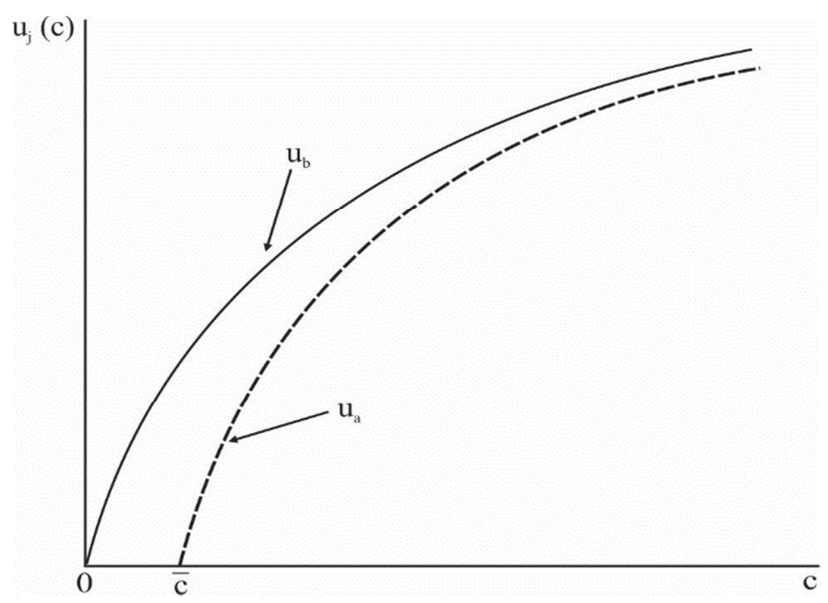

Fig. 1 Utility functions of economies with Stone-Geary $\left(u_{a}\right)$ and homothetic preferences $\left(u_{b}\right)$

As an example, consider the utility function $u_{j}(c)=\sqrt{c-\bar{c}}, j=a, b$, evaluated at the steady state $c=c^{s s}$ and their associated absolute risk aversion measures $A^{j}(c)=$ $-u_{j}^{\prime \prime}\left(c^{s s}\right) / u_{j}^{\prime}\left(c^{s s}\right), j=a, b$, with $j=a$ for Stone-Geary preferences and $j=b$ for homothetic preferences. Clearly, $A^{a}\left(c^{s s}\right)=1 / 2\left(c^{s s}-\bar{c}\right)>1 / 2 c^{s s}=A^{b}\left(c^{s s}\right)$, that is, an economy with Stone-Geary preferences $(\bar{c}>0)$ has a greater absolute risk aversion than an economy with homothetic preferences $(\bar{c}=0)$. Another example would be the utility function given by $u_{j}(c)=c^{1-\sigma} /(1-\sigma), 0<\sigma<1, j=a, b$. Again, at the steady state, one has $A^{a}\left(c^{s s}\right)=\sigma /\left(c^{s s}-\bar{c}\right)>\sigma / c^{s s}=A^{b}\left(c^{s s}\right) .{ }^{13}$

The shape of the policy functions $k^{\prime}=g_{j}(k), j=a, b$, plays an important role in the convergence speed to the steady state and these functions have the following properties under the Assumptions 1 - 5: (i) $g_{j}(k) \geq 0, j=a, b$; this property follows from the fact that $f(k)-k^{\prime} \geq \bar{c} \geq 0, f(0)=0$, and $f(k)>0$ with $k>0$; (ii) they are single-valued for all $k$; (iii) they are non-decreasing; (iv) they are continuous; (v) in the steady-state, we have $k^{s s}=g_{j}\left(k^{s s}\right)$ for both policy functions, $j=a, b$, where $k^{s s}$ is

\footnotetext{
${ }^{13}$ Note that the logarithmic utility function $u(c)=\ln c$ and the exponential utility function $u(c)=1-$ $\exp (-c)$ cannot be used as examples because they violate at least one of the Assumptions adopted in this paper. The logarithmic function, for example, is unbounded below and the exponential utility function violates the Inada condition.
} 
the steady-state capital stock; (vi) they are $C^{1}$; and (vii) there exists $\bar{k}$ such that $g_{j}(k) \leq$ $\bar{k}$ for all $k<\bar{k}, j=a, b$, and $\bar{k}$ exceeds $\left(f^{\prime}\right)^{-1}(1 / \beta) .{ }^{14}$ In Figure 2 we plotted two policy functions taking into account these properties.

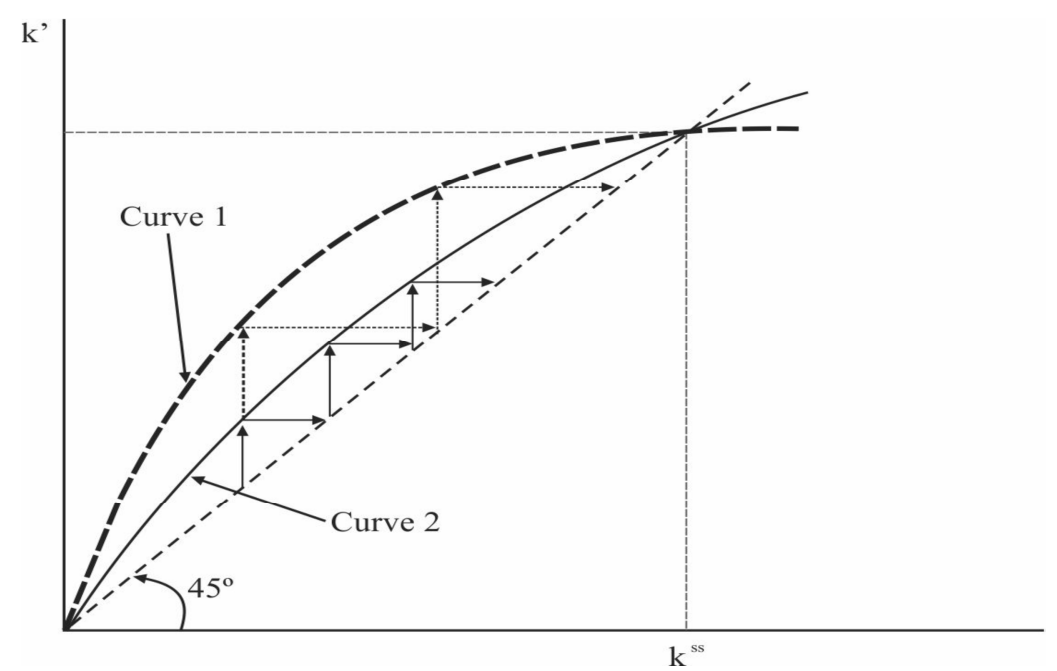

Fig. 2 Two policy functions with different speeds of convergence

As we can see in Figure 2 both policy functions cross the 45-degree line only once, because under the Assumptions 1-5 each optimal sequence of capital stocks is unique and both converge to the same steady-state $k^{s s}$. In this figure, the sequence of capital stocks represented by Curve 1 will approach the steady state faster (that is, in "a smaller number of steps") than Curve 2 and therefore Curve 1 will have a faster convergence speed. We can measure this convergence speeds by looking at their slopes at the steady state, that is, we can linearly approximate these curves around the steady state using first-order Taylor expansions (Krusell 2004). In this sense, taking the firstorder Taylor series expansion of the policy functions we get

$$
\begin{aligned}
& k^{\prime}=g_{j}(k) \approx g_{j}\left(k^{s s}\right)+g_{j}^{\prime}\left(k^{s s}\right)\left(k-k^{\prime}\right), j=a, b, \text { or } \\
& \underbrace{k^{\prime}-k^{s s}}_{\text {Next period's gap }}=g_{j}^{\prime}\left(k^{s s}\right) \underbrace{\left(k-k^{s s}\right)}_{\text {Current gap }}
\end{aligned}
$$

The interpretation is as follows: the closer to zero is the derivative $g_{j}^{\prime}\left(k^{s s}\right)$, $j=a, b$, the faster the convergence will be, that is, the gap is significantly reduced each period. In practice, $g_{j}^{\prime}\left(k^{s s}\right)$ is found by linearizing the Euler equation (7) to obtain a second-order difference equation in $k_{t}$. The smaller of the two eigenvalues of this equation is used as a proxy for convergence speed analysis (see, for example, Krusell

\footnotetext{
${ }^{14}$ For more details on the properties (ii)-(v) of the policy functions, see, for example, Sundaram (1996, p. 301-305); for the property (vi), see Araujo (1991); and for the property (vii), see Krusell (2004).
} 
2004, p. 43-49). Define $\hat{k}_{t}=k_{t}-k^{s s}$. Taking derivatives of the Euler equation (7) with respect to $k_{t}, k_{t+1}$ and $k_{t+2}$, evaluating them at the steady-state $k^{s s}$, and using the fact that $\beta f^{\prime}\left(k^{s s}\right)=1$, we get the expression:

$$
u^{\prime \prime}\left(c^{s s}\right) \hat{k}_{t+2}-\left[u^{\prime \prime}\left(c^{s s}\right)+\frac{u^{\prime \prime}\left(c^{s S}\right)}{\beta}+u^{\prime}\left(c^{s s}\right) \frac{f^{\prime \prime}\left(k^{s S}\right)}{f^{\prime}\left(k^{S S}\right)}\right] \hat{k}_{t+1}+\frac{u^{\prime \prime}\left(c^{s S}\right)}{\beta} \hat{k}_{t}=0 .
$$

After dividing through by $u^{\prime \prime}\left(c^{s s}\right)$, we arrive at the following second-order difference equation:

$$
\hat{k}_{t+2}-\left[1+\frac{1}{\beta}+\frac{u_{j}^{\prime}\left(c^{s S}\right)}{u_{j}^{\prime \prime}\left(c^{S S}\right)} \frac{f^{\prime \prime}\left(k^{s S}\right)}{f^{\prime}\left(k^{S S}\right)}\right] \hat{k}_{t+1}+\frac{1}{\beta} \hat{k}_{t}=0, j=a, b .
$$

Note that the only difference between an economy with Stone-Geary preferences $(j=a)$ and an economy with homothetic preferences $(j=b)$ is given by the parameter $u_{j}^{\prime}\left(c^{s s}\right) / u_{j}^{\prime \prime}\left(c^{s s}\right), j=a, b$, which is the negative inverse of the absolute risk aversion. The magnitude of this parameter will be used to show that a Stone-Geary preference economy will converge more slowly to the steady state than an economy with homothetic preferences. Recall that all other parameters of this difference equation, by hypothesis, are identical for both economies.

Let

$$
\varphi\left(r_{j}\right)=r_{j}^{2}-\left[1+\frac{1}{\beta}+\frac{u_{j}^{\prime}}{u_{j}^{\prime \prime}} \frac{f^{\prime \prime}}{f^{\prime}}\right] r_{j}+\frac{1}{\beta}, j=a, b .
$$

Note that

$$
\varphi(0)=\frac{1}{\beta}>0 \text { and } \varphi(1)=-\frac{u_{j}^{\prime}}{u_{j}^{\prime \prime}} \frac{f^{\prime \prime}}{f^{\prime}}<0, j=a, b .
$$

Since $\varphi(\cdot)$ is a continuous function of $r_{j}$ by the Mean Value Theorem there exists one (root) eigenvalue positive and smaller than 1 . The other eigenvalue must also be positive and larger than 1 since $\lim _{r_{j} \rightarrow \infty} \varphi\left(r_{j}\right)=+\infty>0$ (Krusell, 2004). As already mentioned, the difference in the speed of convergence between economies with StoneGeary preferences and those with homothetic preferences will depend basically on the magnitude of the expression $u_{j}^{\prime}\left(c^{S S}\right) / u_{j}^{\prime \prime}\left(c^{s s}\right), j=a, b$, that will affect, in a differentiated way, the magnitude of the roots of the characteristic equation $\varphi\left(r_{j}\right)=0$.

To see this, consider the following quadratic equation:

$$
r_{j}^{2}-p_{j} r_{j}+q=0, j=a, b \text {. }
$$

Adding $\left(p_{j} / 2\right)^{2}$ and subtracting $q$ from both sides of this equation, we get 


$$
\left[r_{j}-\left(p_{j} / 2\right)\right]^{2}=\left(p_{j} / 2\right)^{2}-q \text { or } r_{j}=\frac{p_{j}}{2} \pm \sqrt{\left(\frac{p_{j}}{2}\right)^{2}-q}
$$

Suppose, without loss of generality, that $p_{a}<p_{b}$. The resulting real roots satisfy the following inequalities: $r_{1 a}>r_{1 b}$ and $r_{2 a}<r_{2 b}$, where the subscript 1 refers to the lowest roots. The second inequality, $r_{2 a}<r_{2 b}$, follows from the fact that

$$
r_{2 a}=\frac{p_{a}}{2}+\sqrt{\left(\frac{p_{a}}{2}\right)^{2}-q}<\frac{p_{b}}{2}+\sqrt{\left(\frac{p_{b}}{2}\right)^{2}-q}=r_{2 b}
$$

since we are assuming that $p_{a}<p_{b}$. The first inequality, $r_{1 a}>r_{1 b}$, follows from a property of the roots of a quadratic equation (equation (8)) that must satisfy $r_{1} \cdot r_{2}=q$. Since $q$ is fixed for both quadratic equations and $r_{2 a}<r_{2 b}$, this implies that $r_{1 a}>r_{1 b}$. These facts are used to show that economies with Stone-Geary preferences converge more slowly to the steady state when compared with economies with homothetic preferences. We just have to show that that the smallest root of the quadratic equation $\varphi\left(r_{j}\right)=0, r_{1 j} \in(0,1)$, with Stone-Geary preferences $(j=a)$ is bigger than the smallest root of this equation with homothetic preferences $(j=b)$, that is, $r_{1 a}>r_{1 b}$.

To see this, let $p_{j}=1+\frac{1}{\beta}+\frac{u_{j}^{\prime}}{u_{j}^{\prime \prime}} \frac{f^{\prime \prime}}{f^{\prime}}, j=a, b$, and $q=\frac{1}{\beta}$. We know from the analysis of absolute risk aversion made above that $u_{a}^{\prime}\left(c^{s S}\right) / u_{a}^{\prime \prime}\left(c^{s s}\right)>u_{b}^{\prime}\left(c^{s s}\right) / u_{b}^{\prime \prime}\left(c^{s s}\right)$. Note that the expression $u_{j}^{\prime}\left(c^{s s}\right) / u_{j}^{\prime \prime}\left(c^{s s}\right)$, $j=a, b$, is negative and in absolute values we have $\left|u_{a}^{\prime}\left(c^{s s}\right) / u_{a}^{\prime \prime}\left(c^{s s}\right)\right|<\left|u_{b}^{\prime}\left(c^{s s}\right) / u_{b}^{\prime \prime}\left(c^{s s}\right)\right|$. When we multiply $u_{j}^{\prime}\left(c^{s s}\right) / u_{j}^{\prime \prime}\left(c^{s s}\right), j=$ $a, b$, by the same negative expression $f^{\prime \prime}\left(k^{s s}\right) / f^{\prime}\left(k^{s s}\right)$ we get as a result that $p_{a}<p_{b}$. Based on the discussion of the roots of the quadratic equation made above, we conclude that $r_{1 a}>r_{1 b}$. These facts and arguments prove the following Proposition:

Proposition 2 Under Assumptions 1-5, economies with Stone-Geary preferences converge more slowly to the steady state equilibrium when compared with economies with homothetic preferences.

The result of Proposition 2 shows that, for economies converging to the same steady state, those with Stone-Geary preferences converge at slower rates when compared to economies with homothetic preferences. Given the optimal sequences of capital stocks $k^{a}=\left(k_{0}, k_{1}^{a}, \ldots, k_{t}^{a}, \ldots\right)$, in the case of Stone-Geary preferences, and $k^{b}=\left(k_{0}, k_{1}^{b}, \ldots, k_{t}^{b}, \ldots\right)$, in the case of homothetic preferences, and the fact the $k_{0} \geq \bar{c} \geq 0$ and $\bar{c} \leq k^{s s}$, the result of Proposition 2 also implies that $k_{t}^{a} \leq k_{t}^{b}, \forall t$, from 
$k_{0}$ to $k^{s s}$, assuming that $k_{0}<k^{s s}$, since the policy functions are non-decreasing. ${ }^{15}$ Although there is no consensus in the empirical economic literature on the speed of convergence between economies, the theoretical result of Proposition 2 is consistent with the hypothesis that less developed economies (at least those with subsistence consumption) tend to converge to the steady-state at slower rates than developed economies. $^{16}$

Our theoretical results should be used with caution when linking them with the existing empirical literature on convergence. Indeed, these results were obtained under very restrictive assumptions of two closed economies that differ only with respect to the preferences structure. Real economies differ not only with respect to preferences, but also with respect to several other factors such as the ability to absorb new technologies and participation in global markets. These other factors were completely ignored in our models of economic growth. However, there is consensus in the literature (see, for example, Steger 2000) that the requirement for minimum consumption actually affects the process of economic growth.

The intuition behind Proposition 2 is simple: part of the capital that could be used in the production process is diverted to ensure a minimum level of consumption, $\bar{c}>0$, in economies with Stone-Geary preferences. Hence, an economy with Stone-Geary preferences tends to save less capital in each period when compared to an economy with homothetic preferences, except at the steady state equilibrium, where both economies save the same amount of capital stock $k^{s s}$. It is interesting to note that if the utility functions were exponential, that is, $u_{j}(c)=1-\exp (\bar{c}-c), \bar{c} \geq 0, j=a, b$, the convergence speed of both economies would be equal. However, as we noted in footnote 13 , the exponential utility function violates Inada condition and because of this there is no guarantee that the intertemporal optimization problem (4) - (5) will have an optimal interior solution that is fundamental to our analysis.

In order to illustrate the magnitude of the difference between the optimal capital dynamic paths under homothetic and Stone-Geary preferences, we arbitrarily use the following functional forms for production function and utility functions $f(k)=k^{\alpha}$, $u_{j}(c)=\sqrt{c-\bar{c}}, j=a, b$, respectively, and we set parameter values as follows: $\alpha=0.33, \beta=0.95$, and $\bar{c}=0.0124$, but it is possible to show that the results are

\footnotetext{
${ }^{15}$ Obviously, if $k_{0}>k^{S S}$, this inequality is reversed, that is, $k_{t}^{a} \geq k_{t}^{b}, \forall t$.

${ }^{16} \mathrm{See}$, for example, Barro and Sala-i-Martin (1992) or de la Fuente (1997) for empirical evidence of this hypothesis.
} 
qualitatively identical under different parametrizations satisfying the assumptions earlier introduced. Given this parameterization and solving for the steady state capital stock, we get $k^{s S}=0.177$ and the corresponding optimal consumption paths are $c_{a}^{s S}=$ 0.3753 and $c_{b}^{S S}=0.3877$. Figure 3 depicts the policy functions under homothetic $(j=b)$ and Stone-Geary $(j=a)$ preferences, clearly showing that they differ and that the optimal capital stock of the economy with homothetic preferences converges more rapidly to the steady state. ${ }^{17}$

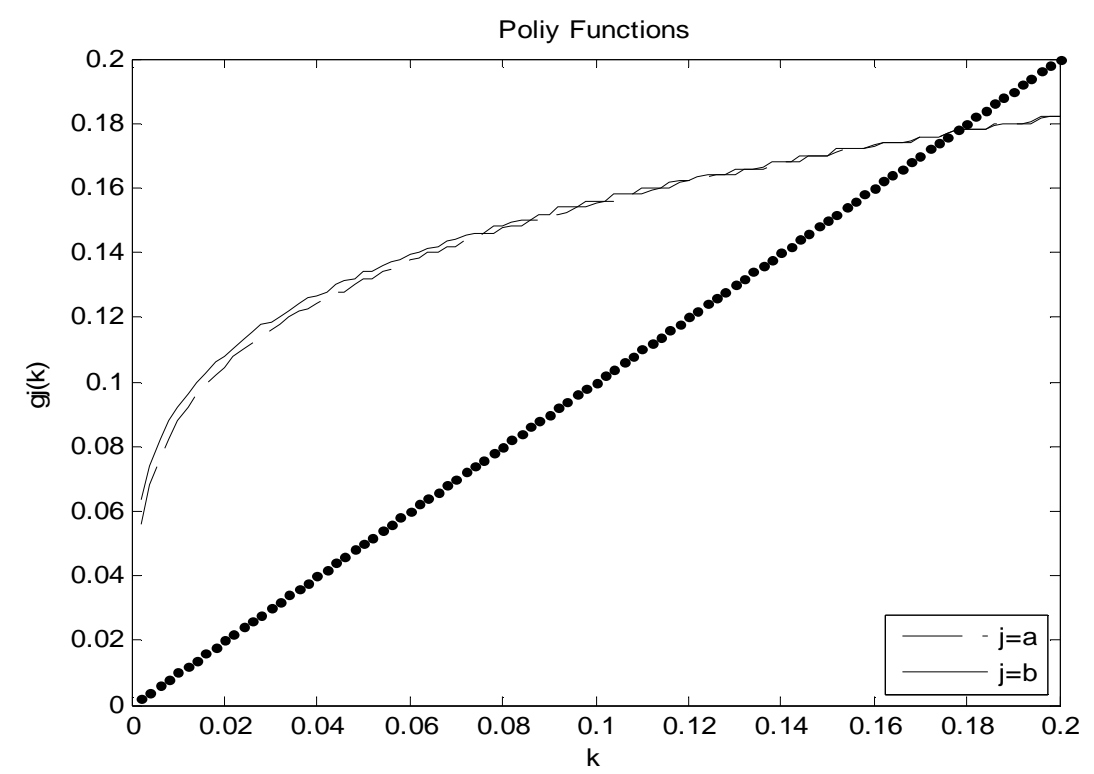

Fig. 3 Policy functions of economies with homothetic $(j=b)$ and Stone-Geary $(j=a)$ preferences

From the parameterization used, we can extract several indicators that confirm our theoretical results. For example, the relationship between the negative inverse of absolute risk aversion between economies with Stone-Geary and homothetic preferences at the steady-state is given by $u_{a}^{\prime} / u_{a}^{\prime \prime}=-0.75064>-0.77544=u_{b}^{\prime} / u_{b}^{\prime \prime}$ and the common parameter $f^{\prime \prime}\left(k^{s s}\right) / f^{\prime}\left(k^{s s}\right)$ is equal to -3.785307 . The quadratic equation of the economy with Stone-Geary preferences is given by $r_{a}^{2}-4.894003$. $r_{a}+1.0526=0$ and the quadratic equation of the economy with homothetic preferences is given by $r_{b}^{2}-4.987878 \cdot r_{b}+1.0526=0$. The corresponding roots are $r_{1 a}=0.225467 ; r_{2 a}=4.668537$ (Stone-Geary preferences) and $r_{1 b}=0.220806$; $r_{2 b}=4.767072$ (homothetic preferences) and, as expected, $r_{1 a}>r_{1 b}$.

\footnotetext{
${ }^{17}$ The Matlab codes that allowed the creation of Figures 3 and 4 were adapted from McCandless (2008, p.67-68) and are in the Appendix B of this paper.
} 
Our next result compares social welfares (measured by the value functions) between economies with Stone-Geary preferences $(j=a)$ and those with homothetic preferences $(j=b)$. Our proof is based on the optimal trajectories of consumption that is given by $c_{t}^{j}=f\left(k_{t}^{j}\right)-k_{t+1}^{j}-\bar{c}, j=a, b, \forall t$, with $\bar{c}=0$ for the economy with homothetic preferences. We show that $v^{a}\left(k_{0}\right)<v^{b}\left(k_{0}\right)$.

Proposition 3 Under Assumptions 1-5, the neoclassical growth model (4)-(5) exhibits different optimal paths for consumption and capital stock, according to the type of preferences used, and these optimal solutions satisfy $v^{a}\left(k_{0}\right)<v^{b}\left(k_{0}\right)$ with $k_{0} \geq \bar{c} \geq 0$ and $\bar{c} \leq k^{s s}$.

Proof See Appendix A

Proposition 3 states that the preferences structure affects the dynamic of both the capital stock and consumption, implying thus that social welfare will also differ according to the type of preferences. In particular, social welfare (measured by the value function) will be highest under preferences of group (b) and lowest under preferences of group (a). This is due to the strictly higher consumption of the representative consumer in the economy with homothetic preferences when compared to the economy with Stone-Geary preferences. This difference in consumption is more evident in the steady state. Therefore, the strictly higher level of consumption in the economy with homothetic preferences will imply a higher level of welfare in this economy.

The difference between levels of welfare in the two economies will depend only on the magnitude of the shift parameter $\bar{c} \geq 0$ that represents the minimum consumption requirement. If $\bar{c} \rightarrow 0$, then $v^{a}\left(k_{0}\right) \rightarrow v^{b}\left(k_{0}\right)$. Note that, for a fixed $\bar{c}>$ 0 , when consumption increases, the difference between utilities (that represent homothetic and Stone-Geary preferences) tends to decrease. With Proposition 1 and 2 we have shown that if two economies have the same technology and the same discount factor, their respective social welfares, capital dynamics and convergence speed to the steady-state can differ in response to changes in representative agent preferences.

Figure 4 illustrates the welfare (values functions) of economies with Stone-Geary and homothetic preferences obtained using our previous parameterization. Note that the difference in welfare levels between economies is sharper than in the case of policy functions (Figure 3). This result clearly illustrates that economies with identical structures, differing only with respect to the structure of preferences, have distinct levels 
of well-being. In particular, economies with homothetic preferences have higher welfare levels than an economy with Stone-Geary preferences.

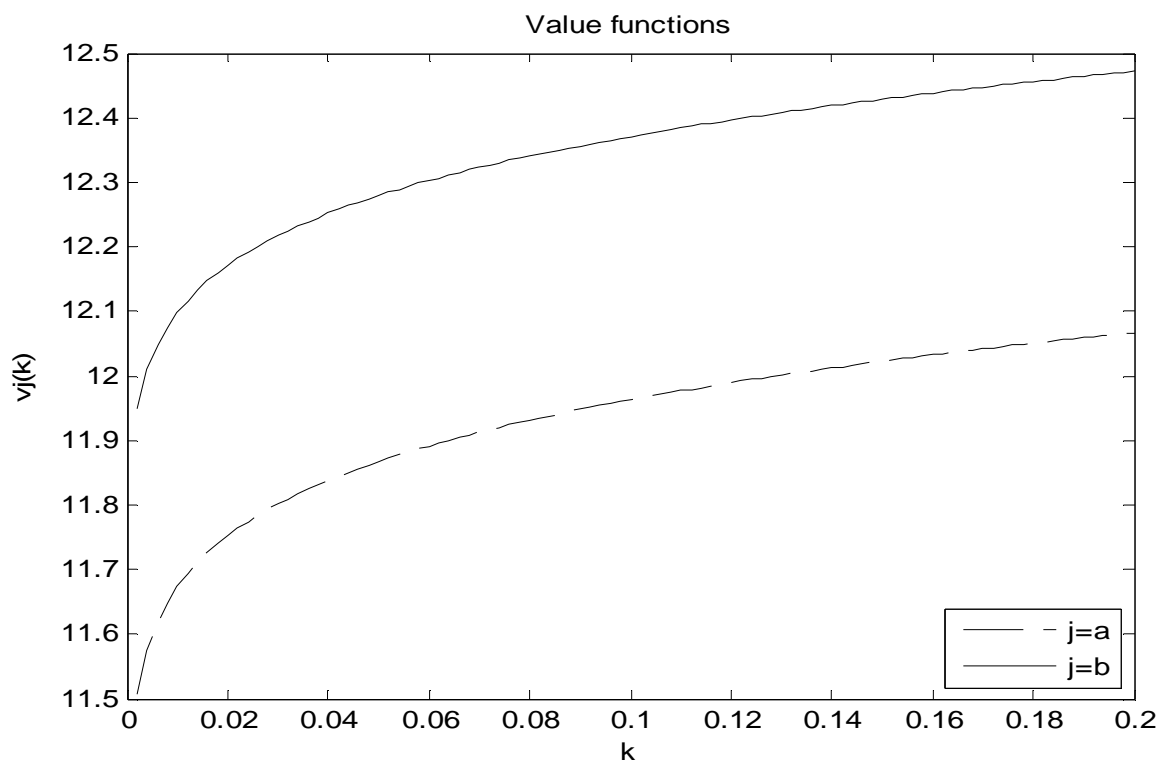

Fig. 4 Value functions of economies with Stone-Geary and homothetic preferences

\section{Conclusions}

In this paper we analyze the effects on welfare, dynamics of capital and convergence to steady state in the Ramsey model under two classes of Gorman preferences: homothetic vs. Stone-Geary (non-homothetic). We show that preferences can affect not only welfare and the optimal path of capital accumulation but also the speed of convergence to the steady state of economies with similar structures. Therefore, even if two economies have the same production technology and the same discount factor and initial capital stock, they may experience different welfare and capital accumulation patterns as well as convergence speed to the steady-state whenever consumers have different preferences. For future research we would like to investigate the effects on the dynamic of capital and speed of convergence to the steady-state of other preference classes and/or consider multi-sectoral models. 


\section{Appendix A: $\quad$ Proof of Propositions 1 and 3}

Proof of Proposition 1: Suppose not, that is, suppose that the optimal sequences of capital stocks of both economies from $k_{0}$ to the single steady-state capital stock $k^{s s}$ are identical. These optimal capital stock sequences must satisfy their respective Euler equations (7), that is, for any $t, t+1$, and $t+2$, we have:

$$
u_{a}^{\prime}\left(f\left(k_{t}\right)-k_{t+1}-\bar{c}\right)=\beta u_{a}^{\prime}\left(f\left(k_{t+1}\right)-k_{t+2}-\bar{c}\right) f^{\prime}\left(k_{t+1}\right)
$$

for the economy with Stone-Geary preferences $(\bar{c}>0)$, and

$$
u_{b}^{\prime}\left(f\left(k_{t}\right)-k_{t+1}\right)=\beta u_{b}^{\prime}\left(f\left(k_{t+1}\right)-k_{t+2}\right) f^{\prime}\left(k_{t+1}\right)
$$

for the economy with homothetic preferences. Since these economies, by Assumption, have the same technology and same discount factor, their identical optimal capital stock trajectories must satisfy:

$$
\begin{gathered}
\frac{u_{a}^{\prime}\left(f\left(k_{0}\right)-k_{1}-\bar{c}\right)}{u_{b}^{\prime}\left(f\left(k_{0}\right)-k_{1}\right)}=\frac{u_{a}^{\prime}\left(f\left(k_{1}\right)-k_{2}-\bar{c}\right)}{u_{b}^{\prime}\left(f\left(k_{1}\right)-k_{2}\right)}=\cdots=\frac{u_{a}^{\prime}\left(f\left(k_{t}\right)-k_{t+1}-\bar{c}\right)}{u_{b}^{\prime}\left(f\left(k_{t}\right)-k_{t+1}\right)}= \\
=\frac{u_{a}^{\prime}\left(f\left(k_{t+1}\right)-k_{t+2}-\bar{c}\right)}{u_{b}^{\prime}\left(f\left(k_{t+1}\right)-k_{t+2}\right)}=\cdots=\frac{u_{a}^{\prime}\left(f\left(k^{s s}\right)-k^{s s}-\bar{c}\right)}{u_{b}^{\prime}\left(f\left(k^{s s}\right)-k^{s s}\right)}
\end{gathered}
$$

This result is true only if the optimal trajectories of capital stocks are constant. As we are admitting that the optimal capital stock trajectories of both economies are identical, this occurs only when $k_{0}=k^{s s}$. Since, by hypothesis, $k_{0} \neq k^{s s}$, we get a contradiction which proves that Proposition 1 is true.

Proof of Proposition 3: The first part of this Proposition follows from Propositions 1 and 2. For the second part, we must show that $v^{a}\left(k_{0}\right)=\sum_{t=0}^{+\infty} \beta^{t} u_{a}\left(c_{t}-\bar{c}\right)<$ $\sum_{t=0}^{+\infty} \beta^{t} u_{b}\left(c_{t}\right)=v^{b}\left(k_{0}\right)$, where $c^{a}=\left(c_{0}^{a}, c_{1}^{a}, \ldots, c_{t}^{a}, \ldots\right)$ represents the optimal consumption trajectory of the economy with Stone-Geary preference, and $c^{b}=$ $\left(c_{0}^{b}, c_{1}^{b}, \ldots, c_{t}^{b}, \ldots\right)$ represents the optimal consumption trajectory of the economy with homothetic preferences. Our proof is by contradiction. Suppose not, that is, the optimal consumption trajectories of both economies are such that $c_{t}^{a}-\bar{c} \geq c_{t}^{b}, \forall t$, which implies $v^{a}\left(k_{0}\right) \geq v^{b}\left(k_{0}\right)$. For $t=0$ and assuming that $k_{0}^{a}=k_{0}^{b}=k_{0}$, we must have $f\left(k_{0}\right)-k_{1}^{a}-\bar{c}=f\left(k_{0}\right)-k_{1}^{b}$, which implies that $k_{1}^{a}<k_{1}^{b}$, since $\bar{c}>0$, and $k_{1}^{a}=$ $k_{1}^{b}-\bar{c}$. For the next period, since the production function is non-decreasing (by Assumption 2) and strictly concave (by Assumption 5), we have that $f\left(k_{1}^{a}\right)<f\left(k_{1}^{b}\right)$ and the relation $f\left(k_{1}^{a}\right)-k_{2}^{a}-\bar{c}=f\left(k_{1}^{b}\right)-k_{2}^{b}$ implies that $k_{2}^{a}<k_{2}^{b}$ and $k_{2}^{a}=k_{2}^{b}-$ $\bar{c}-\left[f\left(k_{1}^{b}\right)-f\left(k_{1}^{a}\right)\right]$, widening the distance between $k_{2}^{a}$ and $k_{2}^{b}$. This pattern is 
repeated in the next period. It follows by induction that the entire optimal sequences of capital stocks follow this pattern, i.e., $k_{t}^{a}<k_{t}^{b}, \forall t$, with $k_{t}^{a}$ moving away from $k_{t}^{b}$ when $t$ tends to infinity. This result contradicts a fundamental property of the optimal solution of the intertemporal optimization problem (4) - (5) that the optimal capital stock sequences of both economies must converge to the same steady state, that is, $k^{s s}$. Note in particular that, at steady state, $c^{s s(a)}=f\left(k^{s s}\right)-k^{s s}-\bar{c}<f\left(k^{s S}\right)-k^{s s}=$

$c^{s s(b)}$, since $\bar{c}>0$, that is, at the steady-state the optimal consumption of a Stone-Geary preference economy is strictly lower than the optimal consumption of a homothetic preference economy. The contradiction obtained and the fact that $c^{s s(a)}<c^{s s(b)}$ establish the desired result, that is,

$$
v^{a}\left(k_{0}\right)=\sum_{t=0}^{+\infty} \beta^{t} u_{a}\left(c_{t}-\bar{c}\right)<v^{b}\left(k_{0}\right)=\sum_{t=0}^{+\infty} \beta^{t} u_{b}\left(c_{t}\right) .
$$

\section{Appendix B: $\quad$ Matlab codes used to create Figures 3 and 4 in the text}

\section{B1. Main program}

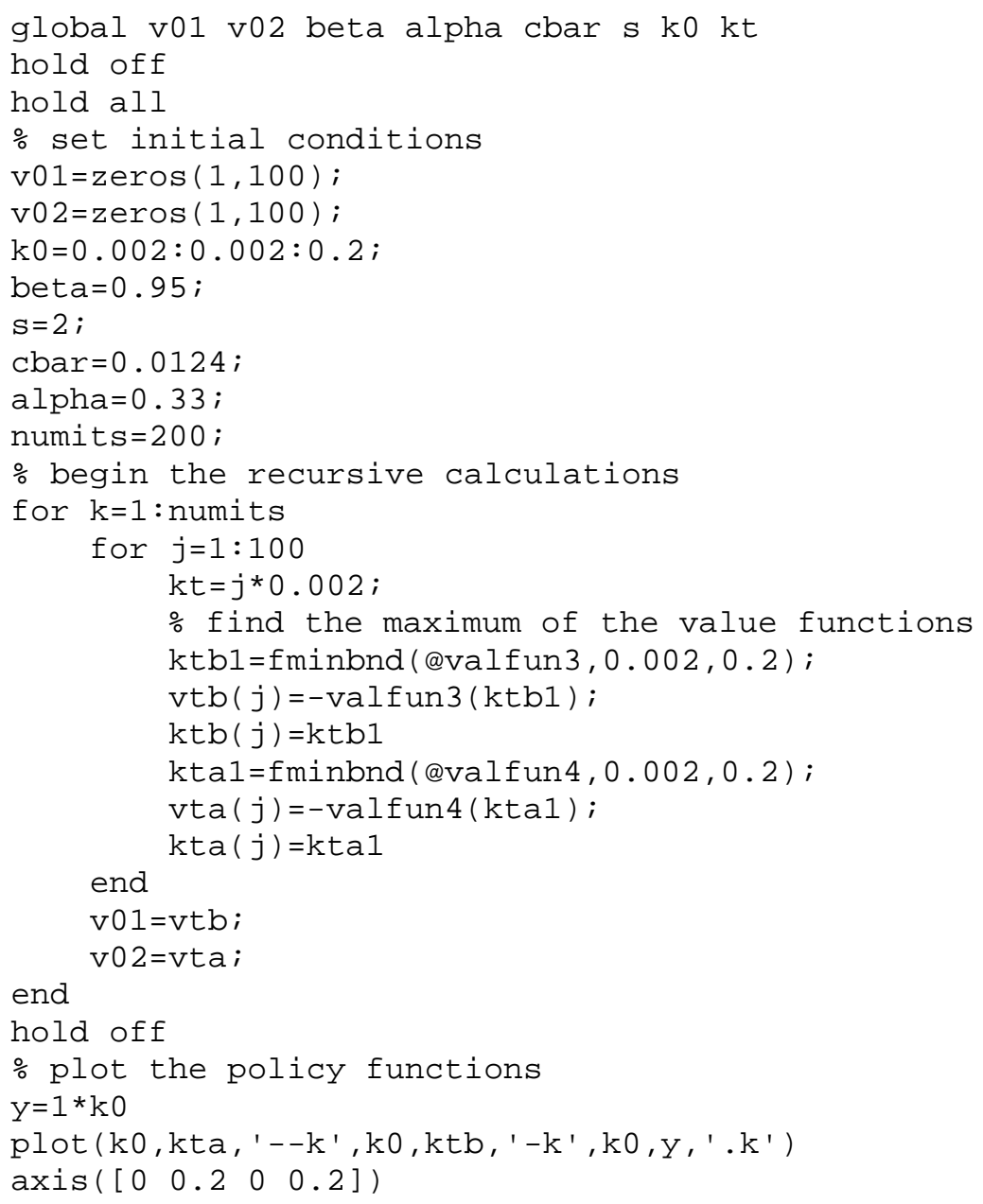




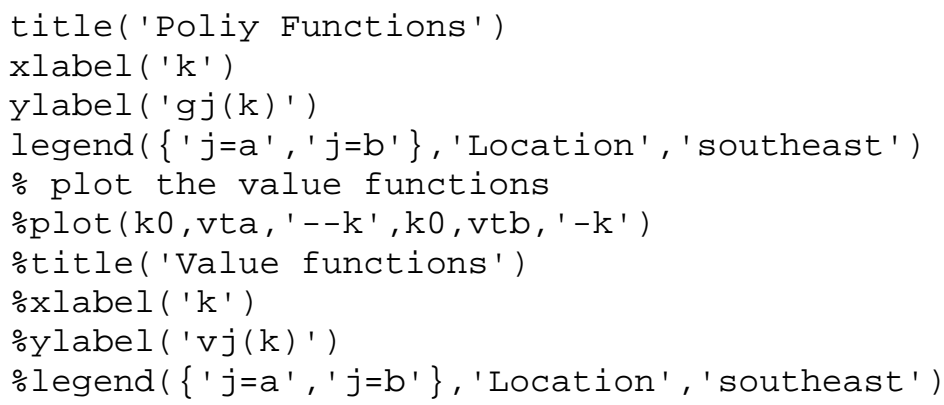

\section{B2. Subroutine (valfun.m) to calculate value functions}

\section{i) Economy with homothetic preferences}

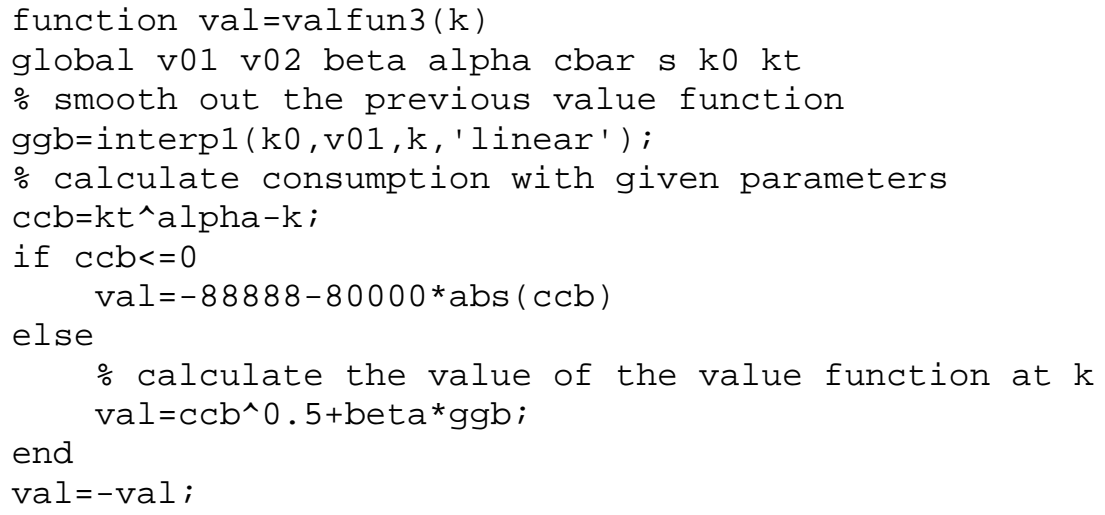

\section{ii) Economy with Stone-Geary preferences}

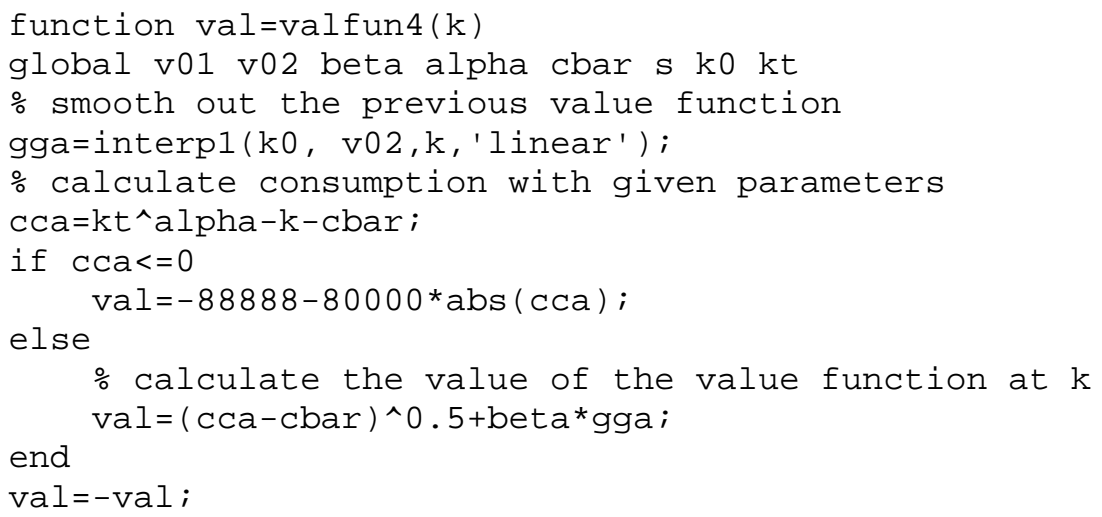

Acknowledgements: We thank the Editor of the Journal and two anonymous reviewers for constructive comments and valuable suggestions. The usual disclaimers apply. 


\section{References}

Álvarez-Peláez MJ, Díaz A (2005) Minimum consumption and transitional dynamics in wealth distribution. Journal of Monetary Economics, 52(3): 633-667

Araujo A (1991) The once but not twice differentiability of the policy function. Econometrica, 59(5): 1383-1393

Atkeson A, Ogaki M (1996) Rate of time preference, intertemporal elasticity of substitution and the level of wealth. The Review of Economics and Statistics, 564572

Barro RJ, Sala-i-Martin X (1992) Convergence. Journal of Political Economy, 100(2): 223-251

Bertola G, Foellmi R, Zweimüller J (2005) Income Distribution in Macroeconomic Models. Princeton University Press, Princeton

Chari VV, Kehoe PJ (2006) Modern macroeconomics in practice: how theory is shaping policy. Journal of Economic Perspectives, 20(4):3-28

Chatterjee S, Ravikumar B (1999) Minimum consumption requirements: theoretical and quantitative implications for growth and distribution. Macroeconomic Dynamics, 3 : 482-505

Chipman, JS (2006) Aggregation and estimation in the theory of demand. History of Political Economy, 38(Suppl_1): 106-129

Crossley TF, Low HW (2011) Is the intertemporal elasticity of substitution constant? Journal of the European Economic Association, 9(1): 87-105

de la Fuente A (1997) The empirics of growth and convergence: a selective review. Journal of Economic Dynamics and Control, 21(1): 23-73

Irz, X, Roe T (2005) Seeds of growth? Agricultural productivity and the transitional dynamics of the Ramsey model. European Review of Agricultural Economics, 32(2):143-165.

Kongsamut P, Rebelo S, Xie D (2001) Beyond balanced growth. The Review of Economic Studies, 68(4):869-882

Krusell P (2004) Lecture Notes for Macroeconomics I. Available at: http://www.econ.yale.edu/smith/econ510a/book.pdf. Accessed 10 June 2019.

Matsuyama K (1992) Agricultural productivity, comparative advantage, and economic growth. Journal of Economic Theory, 58, 317-334

McCandless G (2008) The ABC of RBCs: An Introduction to Dynamic Macroeconomic Models. Harvard University Press, Cambridge

Ortigueira S, Santos MS (1997) On the speed of convergence in endogenous growth models. The American Economic Review, 87(3): 383-399

Pollak, RA (1971) Additive utility functions and linear Engel curves. The Review of Economic Studies, 38(4):401-414

Rubinstein A (2012) Lecture Notes in Microeconomic Theory: The Economic Agent. Princeton University Press, Princeton

Steger TM (2000) Economic growth with subsistence consumption. Journal of Development Economics, 62:343-361

Stokey, NL, Lucas RE with Prescott EC (1989) Recursive Methods in Economic Dynamics. Harvard University Press, Cambridge

Sundaram RK (1996) A First Course in Optimization Theory. Cambridge University Press, Cambridge 